Revue d'histoire de l'Amérique française

RAS REVUE D.HISTOIRE DE L'AMÉRIQUE FRANÇAISE

\title{
À propos de l'École des arts et métiers de Saint-Joachim
}

\section{Lucien Campeau}

Volume 29, numéro 4, mars 1976

URI : https://id.erudit.org/iderudit/303487ar

DOI : https://doi.org/10.7202/303487ar

Aller au sommaire du numéro

Éditeur(s)

Institut d'histoire de l'Amérique française

ISSN

0035-2357 (imprimé)

1492-1383 (numérique)

Découvrir la revue

Citer cette note

Campeau, L. (1976). À propos de l'École des arts et métiers de Saint-Joachim. Revue d'histoire de l'Amérique française, 29(4), 567-570.

https://doi.org/10.7202/303487ar d'utilisation que vous pouvez consulter en ligne.

https://apropos.erudit.org/fr/usagers/politique-dutilisation/ 


\title{
NOTE CRITIQUE
}

\section{À PROPOS DE L'ÉCOLE \\ DES ARTS ET MÉTIERS DE SAINT-JOACHIM*}

\author{
LUCIEN CAMPEAU \\ Département d'bistoire \\ Université de Montréal
}

Je lis dans la livraison de juin 1975 de la Revue un article de Peter N. Moogk intitulé «Réexamen de l'École des Arts et Métiers de Saint-Joachim». Outre que la traduction ne laisse pas toujours le sens transparaître avec clarté, la discussion embrouille beaucoup plus de choses qu'elle n'en éclaire. L'auteur s'empêtre d'abord dans les divers sens du mot "séminaire» (p. 5). Puis, sous prétexte de discussions critiques, il se perd en un tas de considérations confuses sur les intentions des historiens qui ont touché ce sujet. Il fait aussi un usage abusif de l'argument du silence, en une matière où les témoignages ne sont que fragmentaires.

Mon intention n'est que d'indiquer ce que l'auteur aurait pu tirer de ses sources, s'il avait abordé le sujet sans préconception et l'avait traité selon une vraie méthode critique. Le nœud de la question réside dans le témoignage de Denonville, du 15 novembre 1685 (p. 18). Je ne le reproduis pas ici, pour n'être pas trop long.

Ce texte est tout simple. Le premier août 1685 , le gouverneur arrive à Québec par le même navire que Mgr de Saint-Vallier, qui y vient aussi pour la première fois, comme vicaire général de Mgr de Laval. Tous deux trouvent à leur arrivée deux "establissements", deux «maisons» déjà formées «dans le séminaire de l'Evesché». Les deux institutions sont distinctement caractérisées: «...dans l'une, l'on y met ceux auxquels on trouve de la disposition pour les lettres, aux quels on s'atache de les former pour l'Église». Qui ne reconnaîtra pas dans cette description le Petit Séminaire de Québec, fondé

* Voir RHAF, 29, 1 (juin 1975): 3-29.

RHAF, vol. 29, no 4 (mars 1976) 
par Mgr de Laval en 1668? Ce Séminaire est, concrètement, une résidence où les futurs clercs vivent en commun sous la direction de prêtres, s'initiant à la liturgie et à la vie spirituelle, mais suivant les classes de lettres au collège voisin des Jésuites. "Dans l'autre maison on y met ceux qui ne sont propres, que pour estre artisans, et à ceux-là on apprend des mestiers». Si l'on ne peut appeler cette seconde «maison» une école de métiers, il faut renoncer à utiliser les sources en histoire. Deux institutions, chapeautées par le Séminaire de Québec, ayant une existence séparée avant août 1685. Si le lieu du Petit Séminaire, à Québec, ne prête pas à contestation, notre auteur a pris le parti de nier l'existence de l'autre avant la même date. Pourquoi alors deux «establissements», deux «maisons», déjà formés avant l'arrivée de Denonville et Saint-Vallier?

Les indices ne manquent pourtant pas pour localiser l' 'école des métiers» à Saint-Joachim avant 1685 . À partir de cette année, en effet, la situation de l'école à cet endroit ne fait plus problème. En 1677, Mgr de Lavai, propriétaire des fermes du cap Tourmente, les a reprises en main pour exploitation directe, par le moyen d'engagés ou de donnés du Séminaire de Québec, auquel les fermes étaient destinées. C'est la même année 1677 que Louis Soumande fut ordonné prêtre et envoyé presque aussitôt surveiller les fermes tout en faisant le ministère aux environs. Soumande sera avec Mgr de Laval le principal soutien de l'école. En 1678, selon une citation de l'auteur, «J.-B. Lamusette, entré le 8 juillet [au Séminaire], fut envoyé au cap tormente, d'où il sortit ayant appris le métier de masson». On ne peut supposer qu'il a fait son apprentissage sous l'un des maçons habitant la côte de Beaupré. Un membre du Séminaire est sous l'entière dépendance de cette institution, à laquelle il est à charge et entièrement redevable de tout. La soumission à un maître externe, non moins totale, eût été incompatible. En 1681, Claude Denys, fils de Simon, qui a 25 ans et possède une formation littéraire, dirige la principale ferme du Séminaire, assisté d'un vieux couple, Jean Darbois et sa femme. Il a sous son autorité sept jeunes gens: Noël Roy, 20 ans, Jean Allaire, 17 ans, Jean Lamarche, 17 ans, Pierre Deslauriers, 18 ans, François Allaire, 14 ans, Jean Piché, 14 ans, Denis Lavallée, 12 ans. Deux domestiques complètent le personnel. Que signifie cette concentration de jeunes autour d'un homme spécialement qualifié, sinon l'école des métiers que notre auteur n'a aperçue nulle part? Lui-même signale que quatorze garçons ont passé par le cap Tourmente avant 1685 (p. 9). Mais il escamote si bien les faits qu'on ne voit pas l'école. 
Ce qu'il n'a pas compris, mais pas du tout, c'est la nature de l'initiative prise par Mgr de Saint-Vallier en novembre 1685. On sait que le prélat était fécond en idées aventureuses. Il persuada les directeurs du Séminaire d' «établir au Cap tourmente un petit Séminaire d'étudiants, comme celui de Québec». Il ne s'agit plus d'une "école des métiers ", mais d'un dédoublement du petit séminaire de Québec, formé de jeunes gens destinés à la prêtrise. C'était même plus. Alors qu'au Petit Séminaire de la ville, on pouvait se passer de professeurs de lettres, le collège des Jésuites remplissant la fonction de l'enseignement, il en faudrait au cap Tourmente. Ainsi, c'était même un collège qu'entreprenaient de fonder Denonville et SaintVallier. Voilà ce qui était aventureux et ce que Mgr de Laval, alors en France, désapprouva entièrement. Le nouveau "petit séminaire», conjoint avec l'ancienne "école des métiers», est ce que décrit Mgr de Saint-Vallier en 1688: «Mon principal soin dans le Cap Tourmente fut d'examiner l'un après l'autre trente-un enfants que deux ecclésiastiques du Séminaire de Québec y élevaient et dont il y en avait dix-neuf qui s'appliquent aux études, et le reste à des métiers.»

La fondation de ce "petit séminaire d'étudiants" requérait une direction ecclésiastique plus élaborée qu'une simple "école des métiers». M. Louis Soumande, desservant du cap Tourmente, fut nommé directeur et Claude Denys, en place depuis au moins quatre ans, fut revêtu de la soutane et orné du titre de préfet.

Ce qui a été supprimé, vers 1687 , faute de succès et de fonds, ce n'est pas l' «école des métiers», mais le «petit séminaire d'étudiants », dont la plus grande partie furent renvoyés à leurs familles et quatre seulement rapatriés au Séminaire de Québec: La Chesnaye, La Durantaye et les deux frères La Bouteillerie. On n'avait pas trouvé heureuse l'expérience de mêler des «enfants d'études» avec des «enfants de travail». Mais ceux-ci demeurèrent à Saint-Joachim jusque vers 1705, selon M. Honorius Provost (Le Séminaire de Québec, p. 130 n. 5), ou après 1715 selon notre auteur, qui ne donne pas ses raisons.

Ainsi, l' «école des métiers» se trouvait bien à Saint-Joachim avant 1685. Elle donna à Saint-Vallier la pensée d'y adjoindre un «petit séminaire d'étudiants» en cette année, et même à Denonville celle de la transformer en "manufacture» ou atelier de production aussi bien que d'apprentissage. Elle commença au temps où $\mathrm{Mgr}$ de Laval était évêque et même seigneur de Beaupré, seigneurie qu'il céda au Séminaire en 1680. Laval et le Séminaire la soutiendront 
après l'échec des «enfants d'études». Il s'y trouvait une «petite école» - parce que la lecture, l'écriture et le calcul sont utiles aux artisans - alors que le Séminaire n'en avait pas encore à Québec. La seule «petite école» de garçons, en cette ville, se trouvait alors au collège des Jésuites; elle ne sera doublée de celle du Séminaire qu'en 1699. Voilà ce que l'auteur aurait dû voir. 